\title{
Intervención pedagógica para el desarrollo de competencias argumentativas en estudiantes de Educación media
}

\author{
Pedagogical intervention for the development of argumentative skills in \\ middle school students
}

\author{
- CARLOS ANDRÉS TASCÓN ÁLVAREZ \\ carantasal@hotmail.com \\ Código ORCID: 0000-0003-2677-8833 \\ Institución Educativa Técnico Industrial Multipropósito, Colombia
}

Artículo recibido en abril 2019 / Arbitrado en mayo 2019 / Publicado en julio 2019

Resumen

Palabras clave: Comprensión de textos; argumentación; intervención pedagógica; Taller de lectura tendientes a la comprensión y producción de textos..
El presente trabajo se inscribió dentro de las intervenciones destinadas a proveer recursos para desarrollar los procesos de comprensión de textos. Se realizó en una Institución Educativa en Cali, Colombia, con estudiantes de grado Once. A través de un conjunto de estrategias, los participantes pudieron apropiarse de algunas herramientas para la comprensión y discusión del texto argumentativo. El diseño metodológico corresponde a los proyectos especiales, con una propuesta que aspira favorecer la comprensión de las microestructuras del discurso de orden ensayístico; se valió de elementos cuantitativos para la validación previa y de cualitativos para la intervención pedagógica. Los resultados comprobaron que mediante el taller de lectura y análisis, los sujetos mejoraron su comprensión de textos de opinión. El propósito ulterior es continuar con la mediación educativa a través de trabajos de enfoque discursivo que logren guiar al estudiantado en técnicas de argumentación

This work is part of the interventions aimed at providing resources to develop the processes of text comprehension. It was held in an Educational Institution in Cali, Colombia, with eleventh grade students. Through a set of strategies, participants were able to appropriate some tools for understanding and discussing the argumentative text. The methodological design corresponds to the special projects, with a proposal that aspires to favor the understanding of the microstructures of the essayist discourse; it used quantitative elements for prior validation and qualitative elements for pedagogical intervention. The results showed that through the reading and analysis workshop, the subjects improved their understanding of opinion texts. The subsequent purpose is to continue with educational mediation through works of discursive approach that manage to guide students in argumentation techniques aimed at understanding and producing texts..

Keywords: $\quad$ Text comprehension; argumentation; pedagogical intervention; Reading workshop 


\section{INTRODUCCIÓN}

La escuela como centro de cambios sociales positivos debe ser capaz de formar estudiantes autónomos, críticos y dinámicos frente a lo que sucede en su realidad escolar, así como también ante el contexto sociocultural inmediato y el de orden mundial.

En la esfera social el lenguaje se erige como mecanismo de comunicación, de construcción y transformación. Además, permite generar el vínculo para expresar puntos de vista, el análisis de situaciones pragmáticas y difundir el conocimiento de una forma asertiva. La argumentación, por su parte, se convierte en la posibilidad de tomar posiciones reflexivas y críticas de los procesos históricos y culturales de una sociedad.

En concordancia con estos procesos de transformación, en este trabajo se planteó aplicar un conjunto de estrategias con estudiantes de grado Once, del Colegio Multipropósito, Institución Educativa Oficial de Zona de Ladera de Cali, Comuna 20. Se propuso explicar, aplicar y compartir la lectura y comprensión de textos para alcanzar el necesario desarrollo de competencias argumentativas, que les permita organizar sus puntos de vista en relación con lo que sucede en su contexto y establecer diálogos para persuadir o convencer a su interlocutor. El trabajo partió, específicamente, del estudio y comprensión de la organización microestructural de los textos, para propiciar en los jóvenes la comprensión de los discursos argumentativos y con ello una apropiación y crecimiento en el empleo del lenguaje.
Siguiendo a Martínez (2001) y también a Vigotski (1995) se comprendió que el lenguaje se construye de una gran cantidad de variaciones y elementos ricos en su expresión, se constituye en una herramienta para la reflexión interior y con ello el fortalecimiento de la conciencia, a partir de la construcción social y la posibilidad de desarrollar y expresar el pensamiento y sus competencias de argumentación socializadas.

El desarrollo de esas competencias son tarea de la escuela, por lo que la argumentación como acto de habla complejo puede contribuir a la resolución de diferencias de opiniones y a confrontar asertivamente a los otros (van Eemeren y Grootendorst, 2002).

Teniendo en cuenta estos componentes, el proyecto fija su interés en proveer a los estudiantes de herramientas que, en principio, les permita la identificación de cadenas semánticas y precisar la forma cómo el autor privilegia unos términos para la construcción de su punto de vista. De esta forma, cada párrafo se conecta con el anterior y abre el camino discursivo que brinda cohesión y coherencia a todo el escrito (Álvarez, 2007).

En este trabajo, entonces, el objetivo principal fue proponer un taller pedagógico destinado a incrementar la comprensión de las microestructuras del discurso de orden ensayístico en los estudiantes de Grado Once de una Institución Educativa Oficial. Para ello se planteó identificar los tipos de estructuras organizativas del discurso que reconocen los estudiantes y luego de la aplicación de diversos talleres (en este caso se presenta el Taller de Organización Microestructural) 
observar los resultados de la apropiación de nuevas competencias en el desentrañamiento de los significados.

Se aspiró dinamizar mediante las tareas de interpretación y comprensión esa aproximación que debe existir entre autor, texto y lectores propiciada por la argumentación como fundamento de la comprensión de los textos. En la comunicación la figura del "otro" adquiere la de un actor partícipe en la construcción del conocimiento, dotado de la capacidad para hacer del lenguaje la posibilidad de dar significado a la interacción social. En otras palabras, ese significado se elabora por la existencia y la participación de la otredad; por tanto, se puede entender la operatividad del lenguaje, asumido como "la codificación de un «potencial de conducta» en un "potencial del significado», es decir, como un medio de expresar lo que el organismo humano «puede hacer» en interacción con otros organismos humanos, transformándolo en lo que "puede significar»" (Halliday, 1994, p. 33). Se reivindica el aporte de los actores sociales en la significación, tanto de los actos de habla, como en la participación como sujetos lectores y comprensivos.

Por consiguiente, la comprensión de los textos viene dada por un carácter pedagógico que permite la apropiación de unas estrategias para la comprensión de textos. Autor y docente emiten una voz y un sentido que se debe reconocer y reelaborar por parte de los estudiantes, quienes adquieren en el proceso de comprensión de textos las herramientas suficientes que irán desarrollando a lo largo de cada proceso académico, remarcando que, de igual manera, los elementos del discurso también están presentes en la argumentación (Calsamiglia y Tusón Valls, 2002).

El lenguaje, inscrito en un proceso de socialización, va permitiendo definir esos roles en varios espacios de la institucionalidad: la familia, el Estado, la ciudad, la iglesia, los grupos de pares $y$, por supuesto, la escuela. El intercambio de puntos de vista, que han de ser fundamentados en un manejo claro y preciso de la comunicación, así como la comprensión de unos textos determinados y su socialización, es lo que finalmente permitirá producir un discurso orientado no solo a la construcción de conocimientos útiles desde y para la vida cotidiana, sino en la configuración de una identidad y en la formación de ciudadanos y sujetos críticos. Por supuesto, el lenguaje es la base de todo ello, y su comprensión y significación también estarán determinadas por el entorno social en el que se presentan los intercambios comunicativos.

Además, se atendieron los conceptos de géneros discursivos que han de diferenciarse previamente, por cuanto en esta intervención se optó por la argumentación. En ese sentido, al advertir precisamente a la particularidad de los componentes se comprende el concepto de géneros relacionados con la multiplicidad de escenarios en los que interviene un ser humano, con los demás, con su hábitat, consigo mismo cuya dinámica le conducirá a reconocer las variadas formas en las que se pueden expresar desde lo oral y lo escrito:

El común denominador de estos géneros es la necesaria presencia de un interlocutor para que la comunicación incorpore 
efectivamente el sentido y el propósito que se va construyendo desde los acuerdos del contexto compartido, con su componente social indispensable. Baste decir que los enunciados están dirigidos a alguien, así sea que el interlocutor sea la propia persona que los produce. $Y$ que ese interlocutor tiene un bagaje social previo que le permite, bien sea comprender lo que lee o escucha, adhiriéndose a ello o proponiendo otras alternativas de interpretación, o bien producir, asimismo, sus propios textos y enunciados. Esa producción e interlocución propicia, imperiosamente, un diálogo. Y este se ha de orientar hacia la deliberación con el objetivo de que los actores involucrados en la comunicación se encuentren vinculados en una relación que expresa, de manera efectiva, sus lugares de enunciación sin que unos se interpongan a los otros:

El discurso deliberativo hace énfasis en la tonalidad intencional, relacionada más con la búsqueda de los valores y el énfasis en lo aconsejable, lo prudente y lo razonable para establecer una relación dialéctica con el otro, con el fin de lograr una deliberación responsable que lleve a la definición de pautas o reglas que orienten una sociedad democrática. (Martínez, 2005)

Por lo tanto, la comunicación es una acción social con respecto a sus fines, ya que siempre se elabora con una intención y un propósito, pero también de acuerdo con las reacciones que suscite la enunciación. De tal manera que se hace necesario centrarse en los elementos constitutivos del enunciado, como la unidad mínima de análisis en la comunicación, puesto que una elaboración adecuada de los enunciados es un principio para que la comunicación se reafirme en su valor.

Por lo tanto, se consideró la claridad sobre lo que significa la enunciación como una acción, es decir que particularmente necesita de un agente que la haga posible y la desarrolle. En la enunciación se puede notar más el sentido que se le está otorgando a aquello que se comunica, por lo tanto consolida el carácter social de la producción de discurso. Sobre ello Reboul (1986) afirma que

La enunciación es el acto del emisor, del cual resultará el enunciado. Se ha dicho a menudo que la enunciación, hecho psicológico o social, no compete a la lingüística; que pertenece al dominio del "habla", no de la "lengua". Sin embargo, el enunciado puede incluir signos de enunciación, o dicho de otro modo, morfemas que no tienen sentido más que con referencia a su emisor. La enunciación es, pues, un "hecho de la lengua" (p. 85).

Así, la estructura formal de los enunciados posibilita que en un solo género discursivo puedan presentarse varias formas de situación de enunciación, dependiendo de muchos factores; por ejemplo, el público al cual va dirigido.

En estas estructuras organizativas se encuentra la superestructura que alude tanto a su forma como a su fondo, es decir, saber que un texto ostenta la superestructura argumentativa, ya informa que en su contenido hay un diálogo con el lector para persuadirle (Arteaga Quintero y Cova Jaime, 2017). 
Desde luego, esto implica una mirada global de la organización de los textos, pero también comporta entender que las superestructuras facilitan e incluso determinan los esquemas a los que los textos deben adaptarse (van Dijk, 1991). De esa manera Martínez (2002) observa la función de la superestructura de una manera dinámica, en términos de lo que propicia: "La superestructura es una especie de esquema organizacional global al que el contenido del texto (o macroestructura) se adapta" (p. 104).

El molde que presenta la superestructura es una orientación de contenidos, es decir, una cierta capacidad de moverse de manera autónoma dentro de los límites necesarios de dicho esquema. Mientras que las macroestructuras del texto refieren conexiones de carácter global y propician una jerarquía interna de ideas cuya concatenación da sentido a la idea general, las microestructuras incorporan elementos de cohesión (indispensables, a su vez, en la coherencia) que van dando forma al significado global del texto. La cohesión para Calsamiglia y Tusón Valls (2002) es mucho más reconocible en el texto debido a que

constituye una de las más importantes manifestaciones de la coherencia, identificable a partir de elementos lingüísticos visibles y materiales. Se da en el orden interior del texto $y$ funciona como un conjunto de enlaces intertextuales para establecer las relaciones semánticas que precisa un texto para constituirse como unidad de significación. Estos enlaces se establecen tanto en un texto constituido por interlocutores diferentes (en una conversación) como por un mismo locutor (en la exposición de un tema). ( $p$. 230)

Podrían los interlocutores tener diferentes posturas, llegar a acuerdos o complementarse. Además, las ideas deben diferenciarse en las principales -que abren la discusión- y las subordinadas que son aquellas que las alimentan. Cuando se despliegan todos los argumentos en la discusión, esto es, cuando se extienden todos los argumentos para defender un punto de vista, se configura entonces la modelación de un texto discursivo: "en otras palabras, un texto discursivo, o similar, es la suma total de todas las argumentaciones empleadas para defender un punto de vista" (van Eemeren y Grootendorst, 2002, p. 39).

Vistos todos los elementos anteriores, surgen una serie de preguntas relacionadas con las intervenciones pedagógicas en el área de la Enseñanza de la Lengua: ¿Podrían los estudiantes mediante una aplicación estratégica identificar, analizar y comprender los significados de un texto argumentativo? ¿A través de una lectura guiada, para reconocer las microestructuras textuales, a los estudiantes se les facilitaría extraer el significado de un texto discursivo? ¿Podrían llevarse a cabo, sistemáticamente, estas actividades mediante un taller de lectura organizado?

Para dar respuesta a estos interrogantes, en esta intervención se aplica un Taller de estrategias de lectura que es llevado al aula de forma metódica con un texto para cada estudiante con sus respectivas actividades y una guía de orientación para el docente, de tal manera de proceder paso a paso con la 
intervención. En el procedimiento, se utilizaron técnicas de armonización de datos y procesos cualitativos de observación y análisis de materiales, con una metodología secuencial.

\section{MÉTODO}

Las distintas acciones de esta intervención pedagógica se trabajaron bajo el enfoque cualitativo, fundamentalmente porque los resultados se extraen de forma inductiva y no se esperan réplicas exactas en otros grupos sometidos a la misma actividad (Hernández, Fernández-Collado y Baptista, 2006); adicionalmente se utilizaron algunos elementos estadísticos destinados a la validación de instrumentos y procesamiento de exploración inicial. La investigación se llevó a cabo como una investigación de campo y se estructuró como un Proyecto Especial debido a que se presenta un conjunto de estrategias organizadas en un taller dedicado a modificar la realidad inmediata en el aula de clases. La UPEL (2016) afirma que los proyectos especiales incluyen las propuestas de materiales educativos, elaboraciones de producciones tecnológicas y otros productos que lleven a soluciones de problemas conocidos.

Asimismo, Pérez (2016) indica que los proyectos especiales pueden realizarse por etapas: la precisión de la necesidad a intervenir; delineación de la propuesta; descripción del contexto; presentación de normas para clarificar cómo se llevará a cabo la propuesta y su validación; por último realizar una síntesis.

Bajo la modalidad de proyecto especial se puede construir un taller en el que se disponen un conjunto de acciones para intervenir la realidad a partir de una serie de pautas especificadas previamente. Debe tener como requisito, la validación previa por expertos y luego la validación de campo, hasta llegar a una versión final del material, realizar su aplicación y procesar los resultados.

\section{Sujetos de investigación}

En el trabajo los sujetos no fueron elegidos al azar, por lo que se constituyó una población finita de los estudiantes inscritos en el Grado 11-1, de la jornada de la mañana, de la Institución Educativa Técnico Industrial Multipropósito. El grado estaba conformado por un total de 32 estudiantes, 18 hembras y 14 varones, con edades entre los 16 y 18 años. En el proceso de intervención se observaron las características de entrada que se registraron en una lista de ausencias y presencias de rasgos; posteriormente se anotaron las nuevas usanzas que se iban transformando con la práctica en el taller.

\section{Instrumentos y materiales}

Se elaboraron dos instrumentos para aplicarlos a los materiales ya que la validez se estableció a partir del procedimiento de doble validación de Arteaga Quintero (2007). En tal sentido, se elaboró una escala destinada a validar el Taller de Comprensión Microestructural, que fue entregada a cuatro expertos en el área de Investigación Educativa para someter a revisión los cuatro rasgos y los elementos fundamentales del taller: contenido del texto; lenguaje usado; presentación; diseño pedagógico. En ella se presentaba una medición tipo Likert con cinco (5) posibilidades: 
1. Totalmente en desacuerdo

2. En desacuerdo

3. Medianamente de acuerdo

4. De acuerdo

5. Completamente de acuerdo

Posteriormente, se construyó un Taller de Comprensión Microestructural, para ello se eligió el texto "La conspiración de leer" de Sergio Ramírez. Se presentó a dos validadores: la profesora María Cristina Martínez Solís y al profesor Ricardo Salas Moreno.

A continuación se entregó la escala ya validada y el Taller de Comprensión Microestructural a tres expertos en el área de Lengua y Literatura: doctoras Arteaga Quintero, Cova Jaime y Bustamante para que emitieran sus opiniones y con ello proceder a llevar el material al aula, con los estudiantes.

Seguidamente, se mejoró el Taller con la validación para ser llevado al aula de clase. Por último, se elaboró una lista de ausencias y presencias de elementos para ser aplicada a los estudiantes para detectar conducta de inicio.

\section{Procedimiento}

En la ejecución de la intervención pedagógica se elaboraron varios módulos que, de manera secuencial, conducen el trabajo al tema central de la argumentación que permite a los estudiantes ser más analíticos y críticos; en este caso se validó y aplicó el dedicado a la comprensión de estructuras microestructurales.

Se confeccionó una orientación didáctica para el docente, de tal manera que los pasos pudieran sucederse ordenadamente, allí también se expresan las categorías (Cuadro 1) y sus definiciones para precisar los elementos que deberían ser considerados al momento de trabajar con los estudiantes. La ruta a seguir comprendía el trabajo en aula, una presentación interactiva y una explicación de los temas y conceptos, además de la revisión de ejemplos.

Luego, se facilitó, además, una guía para los estudiantes (Cuadro 2) que incluyera los objetivos, una nota sobre el autor y su imagen, el texto y una serie de actividades.

Posteriormente, se revisaron los resultados entregados por los estudiantes y se procedió a cotejar si los sujetos alcanzaron en la práctica las competencias de identificación de las microestructuras que les fueron impartidas, explicadas $y$ ejemplificadas, y si pudieron analizar los temas en cuestión.

Para el docente, la Guía debe suministrar los contenidos que corresponde tener en cuenta para la discusión en clases, primordialmente las categorías relacionadas con la organización textual, no solo las microestructuras, para poder enmarcar las propiedades léxicas del texto en todas sus dimensiones (Cuadro 1).

Hay que recordar que los componentes de la organización de un texto, la microestructura, la macroestructura y la superestructura, están interrelacionados de tal manera que su dependencia es tanto formal como orgánica, por lo que está dotada de una intención y un propósito. Por lo tanto, se propuso una exposición que aborde la superestructura y sus relaciones con la macro y la microestructura (Delicia, 
2011). La estrategia parte de reconocer la superestructura del texto argumentativo, así se comprenderá mejor la dinámica de los otros elementos y su vinculación específica que permite al texto compactarse $y$ ordenarse.

Cuadro 1. Organización estructural del texto

\begin{tabular}{lll}
\hline Categoría & Aspectos & Indicadores \\
\hline Microestructura & - relaciones significativas entre & $\checkmark$ diferentes expresiones para \\
& los términos & referirse a un mismo referente \\
& - un referente preponderante & $\checkmark$ relaciones entre diversos referentes \\
& & $\checkmark$ reiteración semántica \\
Macroestructura & - situación de enunciación & $\checkmark$ disposición pragmática \\
& - significado global del texto & $\checkmark$ punto de enunciación \\
Superestructura & - esquema organizacional & $\checkmark$ introducción jerárquica de ideas \\
& global & $\checkmark$ argumentos \\
& & $\checkmark$ conclusión \\
\hline
\end{tabular}

Se entiende, por consiguiente, que las estructuras textuales (van Dijk, 1980) comprenden la microestructura que se observa cuando el autor de un texto construye relaciones significativas entre los términos y utiliza diferentes expresiones para aludir a un mismo referente o establecer relaciones entre diversos referentes. Es claro que esta estrategia no obedeció a razones puramente mecánicas sino a aspectos tanto semánticos como pragmáticos que permiten que los términos estén conectados significativamente unos con otros.

\section{RESULTADOS}

En principio es perentorio puntualizar que el paradigma de la presente investigación fue el de la comprensión de textos, diferente al de la comprensión de lectura, en tanto que la primera propone como hipótesis el desarrollo de esquemas mentales como la base para el desarrollo cognitivo. Es decir, que si bien es cierto que para el proceso de comprensión e inferencias textuales es necesario tener en cuenta los saberes previos del lector, son las estructuras concebidas las que permiten inferir los esquemas textuales para aprender de los textos. Mientras que la comprensión de lectura parte de la hipótesis que para la comprensión de un texto basta con los saberes previos del lector y el contexto compartido entre este y el texto.

\section{Primeras acciones}

Teniendo en cuenta las características de su población, la estructura de los textos, el modelo de categorías y sus ítems, se elaboró una lista de ausencias y presencias para determinar los elementos de un texto argumentativo reconocidos por los estudiantes; sobre todo de elementos estructurales y noción de género. Se realizó un sondeo, como prueba inicial, de los conocimientos del grupo sobre los rasgos: a) noción de género discursivo; b) reconocimiento de la organización micro y macroestructural del texto; c) reconocimiento de la superestructura textual. Se tomaron en cuenta la actualidad del tema, su pertinencia 
y el posible impacto que pudiera tener sobre el grupo de intervención (con relación a su edad, su contexto y sus cotidianidades, por ejemplo).

Ese acercamiento generó los siguientes resultados:

- En la noción de género discursivo hubo un $72 \%$ de presencia y un $28 \%$ de ausencia de estudiantes que identificaron el género periodístico.

- En la sección de reconocimiento de la organización micro y macroestructural del texto los resultados fueron $49 \%$ de presencia y $51 \%$ de ausencia.

- En el reconocimiento de la superestructura textual de un texto se encontró un $67 \%$ de presencia y un $33 \%$ de ausencia.

Paralelamente, el primer instrumento destinado a la evaluación de la escala de validación del Taller logró un 0,96\% de validez en la totalidad de los indicadores de los cuatro aspectos (contenido, lenguaje usado, presentación, diseño pedagógico). La frecuencia en la escala estuvo marcada por las opciones "de acuerdo" y "completamente de acuerdo".

La validez del propio Taller por parte de los expertos alcanzó un $92 \%$, la deficiencia se encontró en la poca presencia de gráficos, es decir en el rasgo presentación, en donde dos validadores marcaron el rasgo sobre la presencia de imágenes como "medianamente de acuerdo".
La validez de campo se realizó en varios pasos. Se ofreció el Módulo destinado a trabajar la organización microestructural del texto, dividido en dos partes: la primera son las orientaciones para los docentes y la segunda está destinada a los sujetos participantes, cuya presentación se realizó como una guía de trabajo facilitada a los estudiantes ("Taller de comprensión textual", ver Cuadro 2).

\section{Acciones de intervención}

Primera parte para los docentes:

\section{- Módulo: La organización microestructural} del texto

\section{- Sesión: Las relaciones léxicas (Cadenas} semánticas)

- Taller de análisis: Texto: "La conspiración de leer" de Sergio Ramírez (2014)

Se consideró como un texto propicio para la identificación y reconstrucción de cadenas semánticas, puesto que cada párrafo conecta con el anterior y abre el camino discursivo para lo que se generará más adelante. El docente estudia detenidamente las categorías del Cuadro 2.

"La conspiración de leer" invita a retomar los libros, con lo que se pretende reflexionar sobre las prácticas letradas.

La actividad tiene tres objetivos: identificar los elementos microestructurales que garantizan la cohesión local en los textos escritos. Este proceso se desarrolla a partir del reconocimiento de las estructuras de sentido que el autor construye en el desarrollo proposicional de su texto. Se espera que los estudiantes reconozcan las 
cadenas semánticas para la construcción de un escrito cohesivo y coherente; (2) precisar la construcción de cadenas semánticas en un artículo de opinión y (3) analizar la manera como el autor privilegia unos términos (palabras) para la construcción de su punto de vista.

\section{Orientaciones y procedimiento:}

1. Se presenta la guía de trabajo a los estudiantes para que inicien con el proceso de documentación y apropiación de los contenidos de la lectura.

2. El profesor hace una presentación con recursos digitales de las relaciones léxicas de los textos, indica la importancia de la identificación de estas relaciones, dado que los textos son una construcción de ideas con sentido, mas no una sumatoria de ideas o proposiciones aisladas.

3. Se trabajan algunos procedimientos para la construcción de la cohesión léxica en el texto: la reiteración, la sinonimia, la superordinación y la generalización.

4. Por cada uno de estos componentes, se trabaja la definición y precisión en la manera en que aparecen en los textos escritos y un ejemplo para precisar los componentes.

5. Se proyecta el texto "La conspiración de leer", se hace una lectura grupal para que los estudiantes precisen en las cadenas semánticas que se van construyendo y la relación que establecen las palabras en el desarrollo proposicional y entre los párrafos.

6. Se invita a que cada estudiante retome nuevamente la lectura individual del texto para precisar en aspectos relacionados con la comprensión de textos.

7. Se sugiere la necesidad de subrayar los términos que el estudiante considere clave para el reconocimiento de las cadenas semánticas.

8. Se solicita a los estudiantes identificar las cadenas que se construyen a través del texto.

9. Se precisa el punto de vista que construye el autor.

10. Cada estudiante realiza su análisis para ser presentado en la sesión siguiente. 
Cuadro 2 .Taller de comprensión textual

\begin{tabular}{ll}
\hline TALLER DE COMPRENSIÓN & MÓDULO: La organización microestructural de los textos \\
TEXTUAL & Institución Educativa Técnico Industrial Multipropósito \\
& Área: Lengua Castellana - Grado: Once \\
& Docente: Carlos Andrés Tascón Álvarez
\end{tabular}

SESIÓN: Las Relaciones Léxicas

(Organización

microestructural)

TALLER DE ANÁLISIS

Texto: La conspiración de leer

Sergio Ramírez

Eltiempo.com (2014)

Opinión - Columnista
Objetivos:

- Identificar los elementos microestructurales que garantizan la cohesión local en los textos escritos;

- Precisar la construcción de cadenas semánticas en un artículo de opinión y

- Analizar la manera como el autor privilegia unos términos (palabras) para la construcción de su punto de vista

Lea con atención el siguiente texto:

1. Subraye los términos que considere claves para su análisis.

2. Intégrelos estableciendo la o las cadenas semánticas que se han construido en el texto. Diferencie una de otra si es necesario.

3. Según las relaciones entre los términos y las cadenas semánticas que se establecen identifique el punto de vista del enunciador en el texto. Fundamente su respuesta.

4. Identifique y subraye los términos que el autor reitera por repetición.

5. Realice una lista de significados que se reiteran a través de la sinonimia.

6. Por último, enliste los términos utilizados por reiteración superordinación.

7. Redacte un párrafo con las ideas centrales del texto.

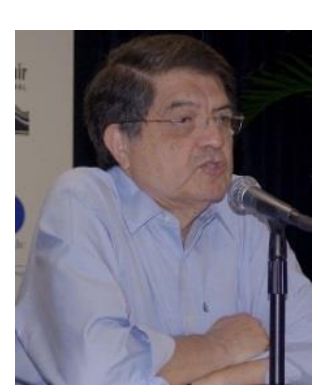

(Nicaragua 5-8-1942) Es un cuentista, novelista, político, ensayista, periodista, abogado, ganador de muchos premios internacionales y un escritor de renombre. Su pensamiento ha sido de gran influencia en el campo de las letras hispánicas y de la cultura en general. Sus trabajos abarcan desde el ensayo periodístico más sencillo, hasta la cinematografía. 
La conspiración de leer

Para que un niño o un adolescente adquieran el vicio de la lectura, antes deben adquirirlo los padres y los maestros, con espiritu cómplice, lejos de la severidad de quien encarga una tarea.

1. Al tratar de iniciar a alguien en la lectura, lo peor es anteponer entre el lector y el libro algún aburrido propósito pedagógico. Un libro sólo es capaz de enseñar si primero gusta. Si no hace reír, si no conmueve, toda enseñanza, toda filosofía se volverán inútiles, pues nadie llega a la última página de un libro fastidioso; y cuando se abandona la lectura al apenas empezar es como si ese libro nunca hubiera sido escrito para quien llegó a tenerlo entre sus manos.

2. Al hablar de la enseñanza de la literatura, Jorge Luis Borges cita una frase del doctor Johnson, el sabio británico de las letras que vivió en el siglo dieciocho: "La idea de la lectura obligatoria es una idea absurda: tanto valdría de hablar de felicidad obligatoria".

3. No hay felicidad obligatoria, pero la lectura depara felicidad; cuando un libro nos atrapa, y llegamos a un punto en que nos sobrecogen el asombro y la admiración, estos sentimientos se transforman en dicha. No podemos sacar gozo del castigo, y un libro impuesto viene a ser un castigo. "Si el relato no los lleva al deseo de saber qué ocurrió después, déjenlo de lado", agrega el doctor Johnson.

4. La Odisea, El Quijote, La Biblia o La Divina Comedia son obras clásicas, y a muchos esa palabra los pone en alerta. A los clásicos, por definición se les considera soporíferos. Al contrario. Un clásico es una promesa de dicha que siempre estará allí.

5. Las novelas no son sobre períodos de la historia, espacios geográficos, teorías filosóficas... Tratan sobre seres como nosotros, sus ambiciones, su idealismo, su perversidad, sus heroísmos y debilidades, la maldad y la nobleza, la generosidad y los celos, y nos muestran cómo estos atributos, siempre en tensión y contradicción, se dan dentro de los mismos individuos.

6. El padre avaro y despiadado que se disputa a la misma mujer con su propio hijo llega hasta nosotros en toda su plenitud en las páginas de Los hermanos Karamazov porque somos capaces de reconocerlo tal como lo retrata Dostoievski; existió, sigue existiendo, así como los muertos de Rulfo que hablan debajo de las tumbas en Pedro Páramo nos son familiares porque lo que cuentan son ambiciones mal cumplidas y pasiones de amor que carcomen hasta en la muerte.

7. No hay que creer a quienes nos dicen que solo debemos aceptar lecturas edificantes, porque así nunca seríamos lectores adictos. Cuántos buenos lectores se han perdido por causa de las imposiciones escolares, que mandan leer libros indigeribles, o que por falta de método son presentados como tales. Y cuántos buenos lectores, y a lo mejor escritores, se han ganado gracias a los libros prohibidos por la escuela, por el hogar, por la religión, porque lo que la imposición no consigue lo consigue la curiosidad por lo prohibido.

8. Y quien no aprende nunca a leer, quien no se vuelve desde temprano un vicioso de los libros, no sabe de lo que se pierde. Se expondrá a llevar una vida mutilada y, a lo mejor, amarga, igual que la de los censores, lejos de los espejismos y los fragores de la imaginación.

9. ¿Cómo crearse ese vicio? Empezando por un cuento de los hermanos Grimm, luego yendo uno de Chéjov, o de Rulfo, antes de llegar por fin a una novela de Faulkner, o al Ulises de Joyce, ya no se diga. O yendo primero a los capítulos y pasajes más divertidos de El Quijote, a alguno de los cuentos de Las mil y una noches.

10. Para que un niño o un adolescente adquieran el vicio de la lectura, antes deben adquirirlo los padres y los maestros, con espíritu cómplice, lejos de la severidad de quien encarga una tarea. Ser parte de la conspiración de leer, comportarse como cabecillas de una hermandad de iniciados. Abrirles una puerta al paraíso, donde espera la manzana dorada entre las frondas del árbol del bien y el mal. 
Luego de la lectura del texto "La conspiración de leer" y la realización de actividades los resultados para presencia y ausencia fueron bastante positivos a causa de las orientaciones previas y el trabajo colaborativo. La identificación del género discursivo, reconocer elementos macro y microestructurales así como precisar la superestructura textual tuvo, gran cantidad de aciertos.

En los análisis presentados por los estudiantes se requería que identificaran, específicamente los indicadores de las microestructuras, de allí que fue preciso que reconocieran las diferentes expresiones para referirse a un mismo referente. Además, se comprobó la cantidad de relaciones que establecieron los estudiantes entre diversos referentes y las reiteraciones semánticas que utilizó el autor. De esta forma, los estudiantes obtuvieron las competencias de lectura para reconocer esos elementos de la microestructura y comentaron la disposición pragmática debido a que se reconocieron como interlocutores del texto.

En sus comentarios precisaron las relaciones léxicas que promovieron en ellos el conocimiento de los textos como una integración. Asimismo subrayaron sinónimos, reiteraciones y pudieron señalar algunos términos que funcionaban en relación semántica por superordinación.

Alcanzaron, asimismo, establecer la progresión temática (Álvarez, 2007; Atienza y López, 1994; Bolívar, 1997) en las cadenas semánticas y en la relación entre proposiciones y párrafos. Finalmente, en la entrega del texto de Sergio Ramírez, los estudiantes subrayaron cadenas léxicas, anotaron elementos relacionados por reiteración y comentaron sus apreciaciones. Con ello se observó la superación de las conductas de entrada de los estudiantes, en el nivel de comprensión estructural, organizativo y argumentativo.

Castillo (2003) precisa que las dificultades en la comprensión se deben a la poca información que tienen los estudiantes sobre los modos de organización discursiva, el desconocimiento de las relaciones micro, macro y superestructurales que se entretejen en los textos y el rol del docente en la enseñanza. Efectivamente, tal como sucede con los estudiantes de la Institución Educativa Técnico Industrial Multipropósito los resultados demostraron la incidencia de la organización de los textos en los procesos de comprensión, las falencias de los estudiantes para determinar las relaciones microestructurales del texto y la poca experiencia de los estudiantes como lectores competentes.

Lógicamente, resalta el rol del docente en la conducción de los estudiantes para identificar las relaciones estructurales de los textos y la necesidad de "favorecer las prácticas de lectura y escritura placenteras (o la lectura y la escritura por el mero placer de leer y escribir), independientes de la lectura y la escritura obligatorias, y/o sin que medien necesariamente otras obligaciones" (Ulloa y Carvajal, 2004, p. 151).

A partir del conocimiento de las categorías de análisis y su localización en los textos, comprendieron el sentido fundamental del escrito. Asimismo, el proceso de intervención planificado por etapas (Ágredo, 2008; Hernández, 2003) contribuyó al cambio positivo en el acercamiento a los textos y la identificación y 
análisis de los elementos microestructurales (Martínez, 2015). En referencia a la compresión textual, es notable el avance de todos los estudiantes entre los resultados de la prueba inicial y la prueba final, lo que reafirma que mediante un trabajo de intervención se puede incidir de manera positiva en la forma como los estudiantes comprenden los textos escritos.

Se pusieron de manifiesto los elementos más relevantes de la argumentación durante la intervención pedagógica, específicamente, el abordaje en el aula del lenguaje como discurso y el aprendizaje desde una perspectiva dialógica (Bajtín, 1984; Ducrot, 1990) que motivó a los estudiantes a aprender de una manera más autónoma y responsable (Perilla, 2006). De igual manera, los trabajos de procesos de comprensión textual fortalecieron esas habilidades en los estudiantes, ad portas de culminar su ciclo en educación media.

También, el aula de clase se convierte en un escenario de discusión, en el que el docente no llega a "vaciar" unos conocimientos en los estudiantes, sino que ellos participan activamente de las relaciones enseñanza y aprendizaje a través de sus aportes, de las discusiones que plantean, de la toma de postura y de las propuestas alrededor de las actividades que deben desarrollar. De igual manera, el fortalecimiento de la lectura crítica se priorizó en este trabajo, tanto porque es invalorable esta habilidad y representa un importante segmento de la calidad de la educación, como para que los futuros ciudadanos conquisten una cultura propia en aquello que se encuentra más allá del texto. Así irán reconociendo lo que a ellos compete como actores sociales, sobre esos temas que directa o indirectamente los afectan y con la potencia de saberse lectores participarán de una trama de complicidades dedicadas a engrandecer la cultura de su nación y su propia ciudadanía, a los efectos de multiplicarla en su entorno inmediato.

\section{CONCLUSIONES}

El rol de los profesores es determinante para incidir de manera positiva sobre los procesos de formación en el aula tanto con unas estrategias pedagógicas adecuadas y novedosas, como con el conocimiento de diferentes teorías para llevarlas al aula de clase en función de las necesidades de sus estudiantes.

En la actividad de evaluación final, hubo un manifiesto desarrollo de las iniciativas de los estudiantes de aproximarse al texto suministrado en el taller, desentrañar la voz enunciativa, identificar sus elementos constitutivos, los lugares de enunciación, entre otros. Todas esas acciones les llevan a desarrollar una competencia analítica y crítica orientada a la elaboración de un criterio propio desde el cual argumentar si se está de acuerdo o no con lo planteado en el

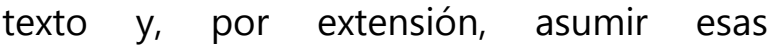
habilidades argumentativas para sí mismos.

Finalmente, el uso del taller en el aula determinó el éxito del trabajo y se evidenció un avance significativo en torno a la comprensión de las microestructuras del artículo de opinión, a la apropiación de las cadenas léxicas y a la identificación de modelos de argumentación, tal como se planteó en el objetivo central. Aun cuando se podría expresar que es necesario insistir en la repetición, aumento y variación de las 
actividades debido a que todavía existen deficiencias en la identificación de algunas relaciones marcadas por la hiperonimia y la superordinación.

Por último, hay que agregar que muchos de los estudiantes después de la experiencia del taller se sintieron motivados a compartir con estudiantes de otros grados, con sus familiares y amigos el contenido del texto leído y su propia experiencia como multiplicadores.

\section{REFERENCIAS}

Ágredo, N. (2008). Incidencia de una Intervención Pedagógica Discursiva e Interactiva en los procesos de Comprensión de Textos Argumentativos en Estudiantes de Octavo de un Colegio Oficial de la Ciudad de Cali. (Tesis de Maestría) Universidad del Valle, Cali, Colombia

Álvarez, L. (2007) Estrategias para el mejoramiento de la enseñanza de la redacción. Caracas: UPEL, Vicerrectorado de Investigación y Postgrado

Arteaga Quintero, M. (2007). Cuentos con ciencia para la infancia de Venezuela. La tecnología multimedia como herramienta de aprendizaje. Revista Investigación y Postgrado, 22(2),11-56

Arteaga Quintero, M. y Cova Jaime, Y. (2017). Comprensión y producción de textos escritos 2. Teoría y Práctica. Caracas, Venezuela: UPEL, Instituto Pedagógico de Miranda José Manuel Siso Martínez

Atienza, E. y López, C. (1994). La progresión temática en el discurso académico. En Llisterri, J.y Poch, D. (eds.) Actas del XII Congreso Nacional de la Asociación Española de Lingüística Aplicada AESLA. Nuevos Horizontes de la Lingüística Aplicada (pp. 33-40). Barcelona: Universitat Autònoma de Barcelona
Bajtín, M. (1984). Estética de la Creación Verbal. México: Siglo XXI Editores

Bolívar, A. (1997). La toma de turnos en el texto escrito: implicaciones para la lectura. En Los procesos de la lectura y la escritura. Cali: Universidad del ValleCátedra UNESCO para la lectura y la escritura

Calsamiglia, H. y Tusón Valls. A. (2002). Las Cosas del Decir. Manual de Análisis del Discurso. Barcelona, España: Editorial Ariel

Castillo, L. C. (2003). Niveles de comprensión y modos de comprender en alumnos de Educación Media. Una evaluación diagnóstica. (Tesis de Maestría), Universidad del Valle, Cali, Colombia

Delicia, D. (2011). Estrategias inferenciales en la comprensión del discurso expositivo: en torno de la adquisición y el desarrollo de las habilidades lingüísticocognitivas. Revista Electrónica de Lingüistica Aplicada, 10, 69-87. Recuperado de http://www.erevistas. csic.es/ficha_articulo.php?url=oai_revista 370:137\&oai_iden=oai_revista370

Ducrot, O. (1990). Polifonía y argumentación: conferencias del seminario Teoría de la Argumentación y Análisis del Discurso. Cali: Universidad del Valle

Halliday, M.A.C. (1994). El Lenguaje como Semiótica Social. La interpretación social del lenguaje y del significado. México: Fondo de Cultura Económica

Hernández, F. (2003). Incidencia de una Intervención Pedagógica en la Comprensión y Producción de Textos Argumentativos Polifónicos desde el Discurso Referido en un grupo de estudiantes de la Universidad del Valle. (Tesis de Maestría), Universidad del Valle, Cali, Colombia

Hernández, R., Fernández-Collado, C. y Baptista. P. (2006). Metodología de la 
Investigación. México: McGraw-Hill Interamericana

Martínez, M.C. (2001). Análisis del Discurso y Práctica Pedagógica. Una propuesta para leer, escribir y aprender mejor. Cali, Colombia: Homo Sapiens Editores

Martínez, M.C. (2002). Estrategias de lectura $y$ escritura de textos. Perspectivas teóricas y talleres. Cali, Colombia: Universidad del Valle-Cátedra UNESCO para la lectura y la escritura

Martínez, M.C. (2005). La construcción del proceso argumentativo en el discurso. Perspectivas teóricas y trabajos prácticos. Cali, Colombia: Universidad del Valle-Cátedra UNESCO para la lectura y la escritura

Martínez, M.C. (2015). Análisis del discurso. Cohesión en español, coherencia y estructura semántica de los textos académicos. Cali, Colombia: Universidad del Valle-Cátedra UNESCO para la lectura y la escritura

Pérez, A. (2016). Proyectos Especiales. Educación y otras ciencias. Recuperado de

http://antonioperezvillegas.blogspot.co m/2012/04/proyectos-especiales.html

Perilla, A. (2006). Evaluación de un Programa de Intervención basado en Estrategias Discursivas Enunciativas en la Comprensión de Textos Expositivos Argumentativos. (Tesis de Maestría) Universidad del Valle Cali, Colombia

Ramírez, S. (2014). La conspiración de leer. EL TIEMPO. Recuperado de http://www.eltiempo.com/archivo/docu mento/CMS-13558741

Reboul, O. (1986). Lenguaje e Ideología. México: Fondo de Cultura Económica

Tascón Álvarez, C. (2016). Incidencia de una propuesta pedagógica enunciativa $y$ dialéctico-crítica del discurso para el desarrollo de la competencia argumentativa en estudiantes de educación media en una Institución Educativa Oficial de ladera de la ciudad de Cali. (Tesis de Maestría) Universidad del Valle, Cali, Colombia

Ulloa, A. y Carvajal, G. (2004). "La Lectura y la Escritura de los estudiantes universitarios: una investigación exploratoria." En: Revista Lenguaje. $\mathrm{N}^{\circ}$ 32. 111-158. Cali: Universidad del Valle UPEL. (2016). Manual de Trabajos de Grado de Especialización y Maestría y Tesis Doctorales. Caracas, Venezuela: Fondo Editorial de la Universidad Pedagógica Experimental Libertador

van Dijk, T. A. (1980). Estructuras y funciones del discurso. Madrid: Siglo XXI

van Dijk, T. (1991). La Ciencia del Texto. Un Enfoque Interdisciplinario. Barcelona, España: Ediciones Paidós

van Eemeren, F. y Grootendorst, R. (2002). Argumentación, Comunicación y Falacias. Santiago de Chile: Universidad Católica de Chile Vygotski, L. (1995). Pensamiento y Lenguaje. Buenos Aires: Ediciones Fausto 